\title{
Metabolomics changes in a rat model of obstructive jaundice: mapping to metabolism of amino acids, carbohydrates and lipids as well as oxidative stress
}

\author{
Yue Long, ${ }^{1,2, \uparrow}$ Xin Dong, ${ }^{3,+}$ Yawei Yuan, ${ }^{1}$ Jinqiang Huang, ${ }^{1}$ Jiangang Song, ${ }^{4}$ Yumin Sun, ${ }^{1}$ Zhijie Lu, ${ }^{1}$ Liqun Yang ${ }^{4}$ \\ and Weifeng $Y^{1}{ }^{1, *}$ \\ 'Department of Anaesthesiology, Eastern Hepatobiliary Surgical Hospital, Second Military Medical University, Shanghai 200438, China \\ 2Department of Anesthesiology, 163th Hospital of PLA, Hunan 410003, China \\ ${ }^{3}$ Department of Pharmaceutical Analysis, School of Pharmacy, Second Military Medical University, Shanghai 200433, China \\ ${ }^{4}$ Department of Anesthesiology, Renji Hospital, School of Medicine, Shanghai Jiao Tong University, Shanghai 200127, China
}

(Received 12 December, 2014; Accepted 4 March, 2015; Published online 4 June, 2015)

The study examined the global metabolic and some biochemical changes in rats with cholestasis induced by bile duct ligation (BDL). Serum samples were collected in male Wistar rats with BDL $(n=8)$ and sham surgery $(n=8)$ at day 3 after surgery for metabolomics analysis using a combination of reversed phase chromatography and hydrophilic interaction chromatography (HILIC) and quadrupole-time-of-flight mass spectrometry (Q-TOF MS). The serum levels of malondialdehyde (MDA), total antioxidative capacity (T-AOC), glutathione (GSH) and glutathione disulfide (GSSG), the activities of superoxide dismutase (SOD) and glutathion peroxidase (GSH-Px) were measured to estimate the oxidative stress state. Key changes after BDL included increased levels of L-phenylalanine, L-glutamate, L-tyrosine, kynurenine, L-lactic acid, LysoPC $(14: 0)$, glycine and succinic acid and decreased levels of Lvaline, $P C^{b}(19: 0 / 0: 0)$, taurine, palmitic acid, L-isoleucine and citric acid metabolism products. And treatment with BDL significantly decreased the levels of GSH, T-AOC as well as SOD, GSH-Px activities, and upregulated MDA levels. The changes could be mapped to metabolism of amino acids and lipids, Krebs cycle and glycolysis, as well as increased oxidative stress and decreased antioxidant capability. Our study indicated that BDL induces major changes in the metabolism of all $\mathbf{3}$ major energy substances, as well as oxidative stress.

Key Words: obstructive jaundice, metabolomics, RPLC-MS, HILIC-MS, oxidative stress

$\mathrm{O}$ bstructive jaundice (OJ) produces profound changes in other organ systems, including the liver, ${ }^{(1)}$ kidneys, ${ }^{(2)}$ heart, ${ }^{(2)}$ brain,$^{(3)}$ blood coagulation and altered body immunity. ${ }^{(4,5)}$ Notably, the incidence of systemic endotoxemia is also increased. ${ }^{(6,7)}$ And the increased oxidative stress in cholestasis may be concerned with the possible mechanism. ${ }^{\left({ }^{(-14)}\right.}$ Metabonomics could provide quantitative measurements of overall alteration in the metabolic profiles of responses to physiopathological stimuli, genetic modification, as well as diseases. ${ }^{(15-26)}$ LC-MS is a frequently-used analytical tools for metabonomic studies and is rapidly expanding in this field. ${ }^{(18,27-29)}$ Recently, ultra-performance liquid chromatography/mass spectrometry (UPLC/MS) has been used broadly in metabolomics researches due to its high sensitivity and reproducibility. ${ }^{(29,30)}$ In the current study, we examined global changes of metabolites after bile duct ligation in rats using a combination of ultra-performance liquid chromatography with Q-TOF mass spectrometry (UPLC/Q-TOF MS) and detected several primary indexes about oxidative stress in serum.

\section{Materials and Methods}

Chemicals and reagents. HPLC-grade methanol and acetonitrile (ACN) were purchased from Merk (Darmstadt, Germany). Formic acid was purchased from Fluka (Buchs, Switzerland). Ammonium formate and all endogenous metabolites related standards were purchased from Sigma-Aldrich (St. Louis, MO). Ultrapure water was prepared with a Milli-Q water purification system (Millipore, Bedford, MA).

Animal experiment and sample collection. All animal studies followed the relevant national legislation and local guidelines. Male Sprague-Dawley rats $(200 \pm 20 \mathrm{~g})$ were purchased from the Slac Laboratory Animal Co., Ltd. (Shanghai, China) and housed in a standard small animal facility. Rats were anesthetized using sodium pentobarbital (Sigma-Aldrich, $50 \mathrm{mg} / \mathrm{kg}$ intraperitoneally, supplemented intravenously as needed). Bile duct was isolated. Then, two 1-0 silk sutures were placed to the proximal portion and one silk suture was placed to the distal part of the bile duct. The silk sutures were tightened. Then the common bile duct was severed. Control rats received sham-operation, but without ligating the bile duct. Blood samples were collected into procoagulant tubes from the hepatic portal vein on the 3rd day after the surgery, left on ice for $1 \mathrm{~h}$, and then centrifuged to obtain serum samples for storage at $-80^{\circ} \mathrm{C}$ until analysis.

Chromatography. Serum samples were thawed at $4^{\circ} \mathrm{C}$, and diluted with methanol at a ratio of $1: 3$. After 3-min vortex, the samples were centrifuged at $12,000 \mathrm{~g}$ for $10 \mathrm{~min}$ at $4^{\circ} \mathrm{C}$. The supernatant was separated with chromatography using an Agilent 1290 Infinity UHPLC system (Agilent Technologies, Wilmington, DE). The column oven was set at $40^{\circ} \mathrm{C}$. An acquity UPLC ${ }^{\circledR}$ HSS T3 $\mathrm{C}_{18}$ column $(1.8 \mu \mathrm{m} 100 \times 2.1 \mathrm{~mm}$, Waters, Milford, MA $)$ was used for the reverse phase separation. The mobile phase consisted of $0.1 \%$ formic acid (A) and acetonitrile modified with $0.1 \%$ formic acid (B), using a gradient elution of $2 \% \mathrm{~B}$ at $0-2 \mathrm{~min}$, $2-95 \% \mathrm{~B}$ at $2-17 \mathrm{~min}, 95 \% \mathrm{~B}$ at $17-19 \mathrm{~min}$. The total run time was 25 min including 6 -min equilibration. The flow rate was

These authors contributed equally to this work

*To whom correspondence should be addressed.

E-mail:ywf808@yeah.net 
$400 \mu \mathrm{l} / \mathrm{min}$ and the injection volume was $4 \mu \mathrm{l}$. For HILIC analysis, an acquity UPLC $^{\circledR}$ BEH HILIC $C_{18}$ column $(1.7 \mu \mathrm{m} 100 \times 2.1 \mathrm{~mm}$, Waters) was used on the same LC system. The mobile phase consisted of $10 \mathrm{mM}$ ammonium formate modified with $0.1 \%$ formic acid (A) and ACN modified with $0.1 \%$ formic acid (B), using a gradient elution of $95 \% \mathrm{~B}$ at $0-10 \mathrm{~min}, 95-90 \% \mathrm{~B}$ at $10-14 \mathrm{~min}, 90 \%$ B at $14-20 \mathrm{~min}, 90-60 \%$ B at $20-23 \mathrm{~min}, 60 \%$ $\mathrm{B}$ at $23-25 \mathrm{~min}$ and post time was set to $10 \mathrm{~min}$ for equilibrating the system. The total run time was $35 \mathrm{~min}$. The flow rate was $350 \mu \mathrm{l} / \mathrm{min}$ and the injection volume was $4 \mu \mathrm{l}$.

Mass spectrometry. An Agilent 6538 UHD and AccurateMass (Q-TOF) mass spectrometer (Agilent Technologies) was used in the study. The Q-TOF mass spectrometer was operated in positive ion mode and negative ion mode with a capillary voltage of $4 \mathrm{kV}$ in positive ion mode and $3.5 \mathrm{kV}$ in negative ion mode, drying gas flow of $11 \mathrm{~L} / \mathrm{min}$, and a gas temperature of $350^{\circ} \mathrm{C}$. The nebulizer pressure was set at $45 \mathrm{psig}$. The fragment or voltage was set at $120 \mathrm{~V}$ and skimmer voltage was set at $60 \mathrm{~V}$. All analyses were obtained by means of an automated calibrant delivery system using a dual-nebulizer ESI source that introduces a low flow $(100 \mathrm{ml} / \mathrm{min})$ of a reference solution (Agilent Technologies), which contains the internal reference masses at $\mathrm{m} / \mathrm{z} 121.0509$, 922.0098 in positive ion mode and $\mathrm{m} / \mathrm{z} 112.9856,1033.9881$ in negative ion mode to ensure mass accuracy and reproducibility. Data were collected in centroid mode and the mass range was set at $\mathrm{m} / \mathrm{z}$ 100-1,100 using extended dynamic range. Potential biomarkers were analyzed by MS/MS. The collision energy was $10-40 \mathrm{~V}$. The negative ion scan was only employed when metabolite identification was carried out.

Measurement of indexes about oxidative stress in serum. The content of malondialdehyde (MDA), superoxide dismutase (SOD), glutathione (GSH), glutathione peroxidase (GSH-Px), oxidized glutathione (GSSG) and total antioxidant capacity (A-TOC) in serum was detected with reagents kits purchased from Jiancheng Biologic Company (Nanjing, China). All manipulations were carried out according to the manufacturer's instructions.

Data handling. In both RPLC (reversed phase chromatography)-MS and HILIC-MS analyses, the raw data obtained in instrument specific format (.d) were converted to common data format (.mzData) files using Agilent Mass Hunter Qualitative Analysis (B.03.01) software that eliminated isotope interferences. The program XCMS (http://metlin.scripps.edu/download/) was used for extracting raw data signals (peak Identification and Integration), and then for correction of retention time and automatic integration and deconvolution analysis (vest MS fragmentation) in $\mathrm{R}$ software platform. The post-editing was conducted in the EXCEL software, and the results were organized as two-dimensional data matrix that included variables (retention time and mass-to-charge ratio), observables (samples) and peak intensity. All data of each sample were normalized to total intensity for correcting the MS response shift during the long analysis duration and the different enrichment factors of serum among individuals before the multivariate data analysis. The normalized data were introduced to SIMCA-P V11.0 (Umetrics, Sweden) for principal component analysis (PCA) and partial least-squares discriminant analysis (PLS-DA) after mean-centering and pareto scaling, a technique that increases the importance of low abundance ions without significant amplification of noise. Different metabolites were selected by using VIP (variable importance in the projection) value (VIP $>1$ was considered statistically significant) and Student's $t$ test $(p<0.05$ was considered statistically significant). Identification of metabolites were conducted by searching online database (http://metlin.scripps.edu/) (comparing the mass-to-charge ratio of Mass spectrum or accurate molecular mass) and comparing with standard compounds. To examine the stability of the LC-MS system and method, quality control (QC) samples were prepared by pooling the same volume of serum from all samples.

We detected several indexes about oxidative stress between the sham group and BDL group. The statistical analysis was carried out using SPSS ver. 17.0 software. Data were given as mean \pm standard error (SE). And differences between two groups were assessed using Independent-Samples $t$ test. It is considered significant if the $p$ value was less than 0.05 .

\section{Results}

Representative RPLC-MS base peak chromatograms (BPC) and HILIC-MS BPC of sham controls are shown in Fig. 1 and 2.

Serum metabolite profile: comparison of sham control vs BDL rats. Data processing using the default Unit Variance Scaling and Mean-Centered for produced a mathematical model, characterized below: $\mathrm{R}^{2} \mathrm{X}=0.668 ; \mathrm{Q}^{2}(\mathrm{cum})=0.499$, indicating a difference in metabolic profile between the sham control and BDL rats. The PCA scores plot (Fig. 3A) showed that all samples were in the $95 \%$ confidence interval, which showed that all samples were in line with the analytical requirements of metabolomics and the BDL 3d group and sham group can also be well distinguished.

Upon verification with partial least-squares discriminant analysis (PLS-DA), the $\mathrm{R}^{2} \mathrm{X}$ (cum) and $\mathrm{R}^{2} \mathrm{Y}$ (cum) values were 0.713 and 0.988 , respectively, indicating goodness of fit and predictive ability. The PLS-DA scores plot (Fig. 3B) also clearly distinguished between the BDL group and sham control group: the BDL group was predominantly in the right side of the first principal component whereas the sham control group was mostly in the left side.

Identification of potential biomarkers. The S-plot (Fig. 3C for RPLC) was used to identify potential biomarkers for obstructive jaundice. A combination of VIP (variable importance in the projection) value of the PLS-DA model with 2-sample Student's $t$ test were used to search differential metabolites between the sham control and the BDL rats, and VIP value $>1, p<0.05$ were considered statistically significant. Candidate metabolites were verified by comparing with online database (http://metlin.scripps. edu/) (comparing the mass-to-charge ratio of Mass spectrum or accurate molecular mass) and comparison using a standard compound. A total of 14 metabolites were differentially expressed (Table 1).

The relative peak areas of the 14 metabolites from both RPLCMS and HILIC-MS to their respective total integrated area of the spectra are shown in Fig. 4. The following eight metabolites were significantly higher in the BDL rats: L-phenylalanine, Lglutamate, L-tyrosine, kynurenine, L-lactic acid, LysoPC ${ }^{\mathrm{c}}$ (14:0), L-alanine, glycine and succinic acid. Six of fourteen metabolites including L-isoleucine, L-valine, $\mathrm{PC}^{\mathrm{b}}$ (19:0/0:0), taurine, palmitic acid and citric acid were significantly decreased. These differentially expressed metabolites were mapped into 4 pathways: amino acid metabolism, lipid metabolism, Krebs cycle and glycolysis (Table 1). A metabolic network is shown in Fig. 5.

Average indices of oxidative stress observed. The primary indexes about oxidative stress were listed in Table 2 . The values of GSSG in rats with BDL were higher than sham control rats, but there was no significant difference between the two groups $(p>0.05)$. MDA and GSH concentrations in rats with BDL were higher than the sham control rats $(p<0.01)$. And the levels of SOD, GSH-Px and total antioxidative capacity (T-AOC) in the BDL group were lower than in the sham group $(p<0.05)$.

\section{Discussion}

As shown in results, HILIC is an appropriate supplementary method for retaining polar metabolites in serum, although it appears to be less effective than RPLC. For instance, L-isoleucine, kynurenine and L-lactic acid were eluted within the dead time on an RP column (see Table 1 for data), while they had longer 

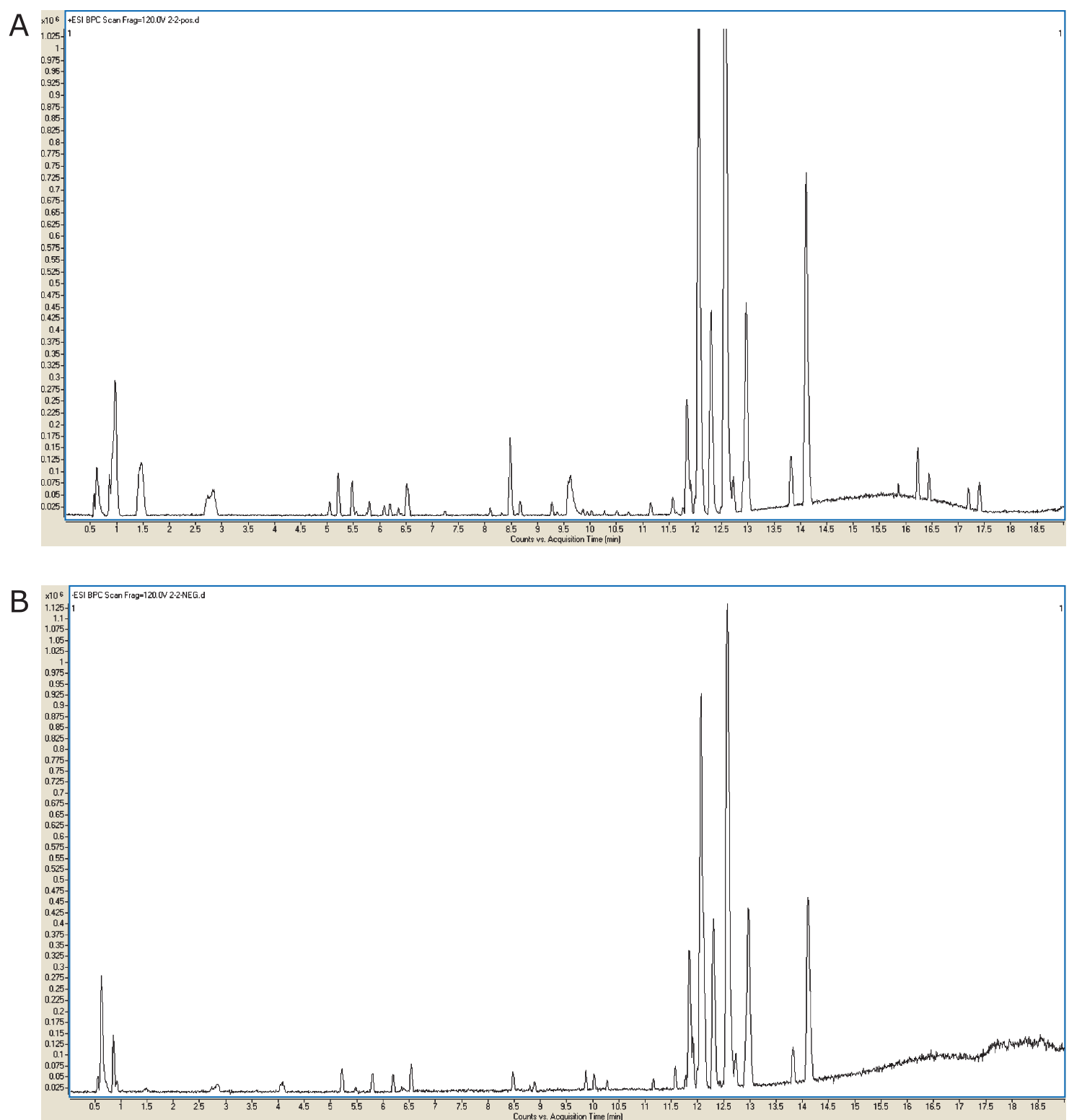

Fig. 1. Representative serum RPLC-MS base peak chromatograms (BPC) of sham rats in (A) ESI+ mode and (B) ESI- mode.

retention times on an HILIC column. It is apparent that HILIC provided higher MS sensitivity because of its enhancive ionization e? ciency in ESI-MS rooting in the high organic content of the solvents used for chromatography. The ions from the polar metabolites with lower concentration could be tested on account of their high sensitivity in HILIC. Hence the units of two chromatographic approaches could add more information about metabolites for the subsequent research.

The current study indicated that metabolic disturbance in BDL rats involves many major biochemical processes. The most prominent changes were amino acids, including L-phenylalanine, L-glutamate, and L-tyrosine. This is not surprising since the liver is the major site for protein metabolism.

Amino acid metabolism. In our experiment, glycine (glucogenic amino acid) was upregulated in BDL rats. Glycine is an antioxidant, and has been shown to be reno-protective via $\mathrm{Na}^{+}-$ $\mathrm{K}^{+}$ATPase, malondialdehyde and nitric oxide. ${ }^{(31)}$ This result encourages exploring glycine as an adjunct treatment of renal failure in patients with cholestasis.
In the recent period, a growing body of research has shown that free glycine is hepato-protective. ${ }^{(32,33)}$ Glycine hinders the increase of $\left[\left(\mathrm{Ca}^{2+}\right) \mathrm{i}\right]$ in hepatic parenchymal cells owing to agonists released during stress, most probably through actions on a glycinesensitive anion channel. ${ }^{(34)}$ Endotoxin activates the cell membrane calcium channels of mononuclear macrophage, increases inward flow of calcium ion, and aggravates the release of cytokines and inflammatory mediators. A previous study showed that glycine could attenuate injury and mortality of endotoxemia via preventing the LPS-induced enhancement of $\left[\left(\mathrm{Ca}^{2+}\right) \mathrm{i}\right]$ in Kupffer cells, and consequently minimizing toxic eicosanoid and cytokine production. ${ }^{(35)}$

L-Glutamic acid and glycine are the rate-limiting precursors in the synthesis of glutathione, and more than $98 \%$ of intracellular $\mathrm{GSH}^{(36)} \mathrm{GSH}$ is very important in protecting against oxidative damage. ${ }^{(37,38)}$ The liver is the major site for detoxicating xenocompounds and ultimately excretion of the substances through the bile. GSH is a main driving force of bile acid independent bile flow and can remove harmful substances via promoting bile 


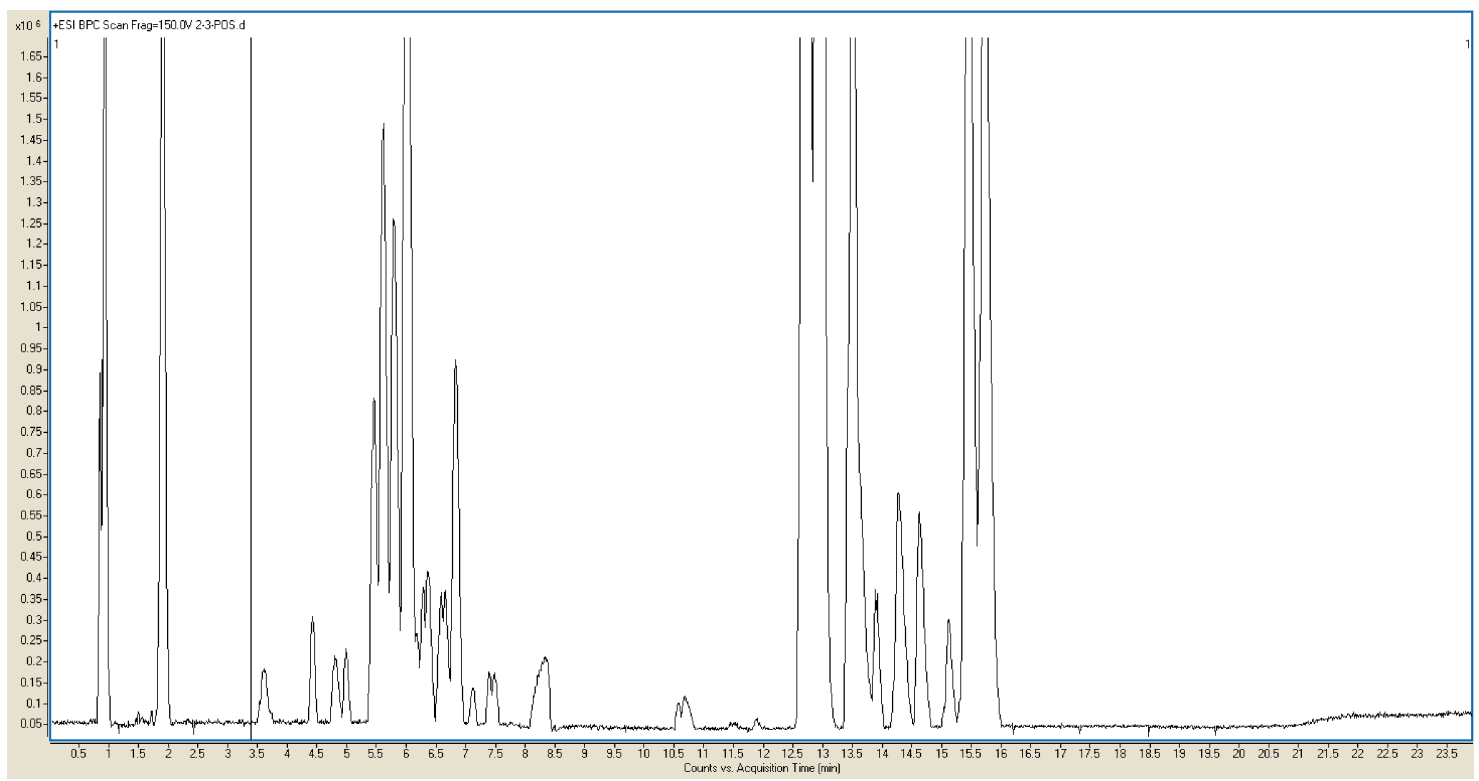

Fig. 2. Representative serum HILIC-MS base peak chromatograms (BPC) of sham rats in ESI+ mode.

A

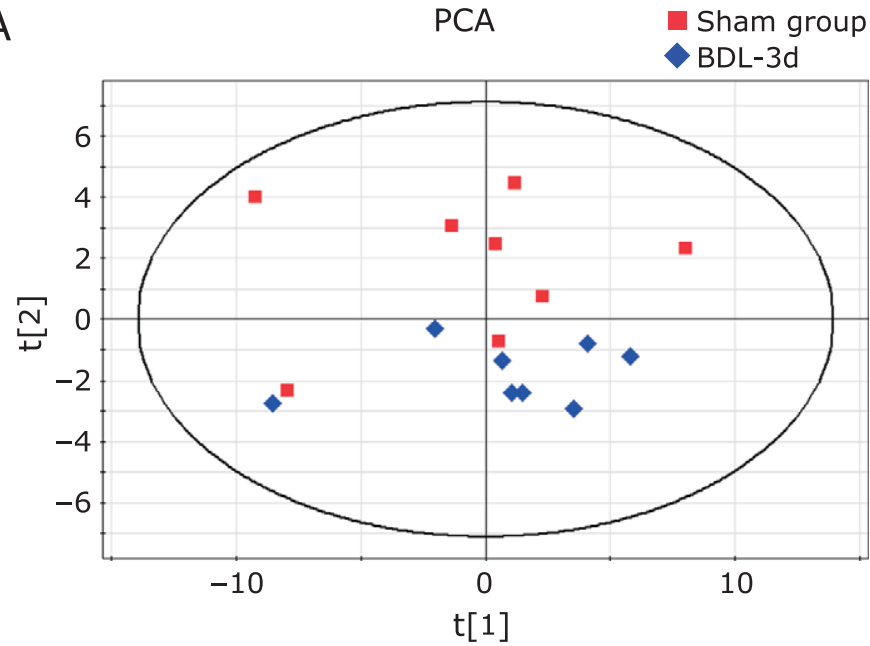

C

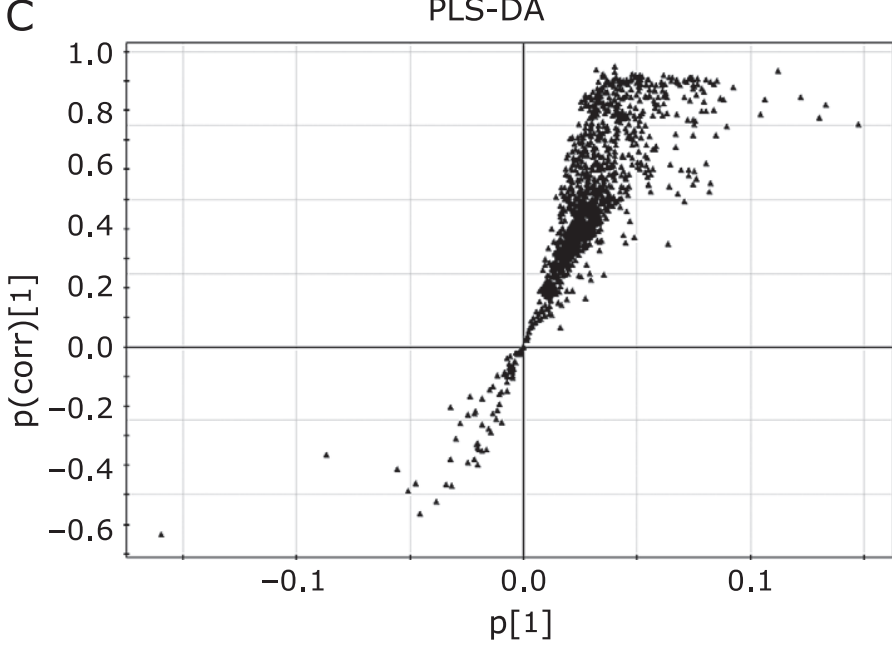

B PLS-DA Sham group BDL-3d

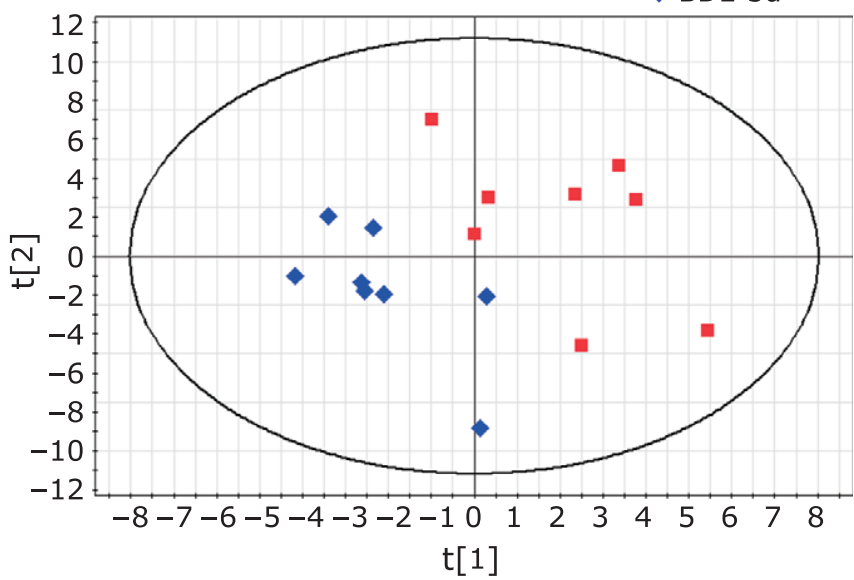

S-plot ( $\square$ ) sham control, $(\diamond) \mathrm{BDL}$. 
Table 1. Potential biomarkers and their metabolic pathways

\begin{tabular}{|c|c|c|c|c|c|c|c|c|}
\hline \multirow{2}{*}{ No. } & \multicolumn{2}{|c|}{ RT (min) } & \multirow{2}{*}{$\mathrm{m} / \mathrm{z}$} & \multirow{2}{*}{ Selected ion } & \multirow{2}{*}{ Formula } & \multirow{2}{*}{ Metabolite } & \multirow{2}{*}{ Trend $^{a}$} & \multirow{2}{*}{ Pathway } \\
\hline & $\mathrm{RP}$ & HILIC & & & & & & \\
\hline 1 & 1.96 & 14.67 & 166.0872 & {$[\mathrm{M}+\mathrm{H}]+$} & $\mathrm{C} 9 \mathrm{H} 11 \mathrm{NO} 2$ & L-Phenylalanine & $\uparrow * * *$ & Amino acid metabolism \\
\hline 3 & 0.68 & - & 148.06 & {$[\mathrm{M}+\mathrm{H}]+$} & $\mathrm{C} 5 \mathrm{H} 9 \mathrm{NO} 4$ & L-Glutamate & $\uparrow * * *$ & Amino acid metabolism \\
\hline 4 & & -15.79 & 118.0863 & {$[\mathrm{M}+\mathrm{H}]+$} & $\mathrm{C} 5 \mathrm{H} 11 \mathrm{NO} 2$ & L-Valine & $\downarrow * \star$ & Amino acid metabolism \\
\hline 5 & 1.03 & 15.23 & 182.0816 & {$[\mathrm{M}+\mathrm{H}]+$} & $\mathrm{C} 9 \mathrm{H} 11 \mathrm{NO} 3$ & L-Tyrosine & $\uparrow * *$ & Amino acid metabolism \\
\hline 8 & 15.46 & - & 538.3887 & {$[\mathrm{M}+\mathrm{H}]+$} & C27H56NO7P & $P C^{b}(19: 0 / 0: 0)$ & $\downarrow * * *$ & Lipid metabolism \\
\hline 9 & 11.76 & - & 468.3104 & {$[\mathrm{M}+\mathrm{H}]+$} & $\mathrm{C} 22 \mathrm{H} 46 \mathrm{NO} 7 \mathrm{P}$ & LysoPCc (14:0) & $\uparrow *$ & Lipid metabolism \\
\hline \multicolumn{9}{|l|}{ ESI- } \\
\hline 10 & 0.69 & - & 124.0076 & [M-H]- & $\mathrm{C} 2 \mathrm{H} 7 \mathrm{NO} 3 \mathrm{~S}$ & Taurine & $\downarrow * * *$ & Amino acid metabolism \\
\hline 11 & 1.11 & - & 120.0295 & {$[\mathrm{M}+\mathrm{FA}-\mathrm{H}]-$} & $\mathrm{C} 2 \mathrm{H} 5 \mathrm{NO} 2$ & Glycine & $\uparrow *$ & Amino acid metabolism \\
\hline 12 & 8.91 & - & 255.2318 & {$[\mathrm{M}-\mathrm{H}]-$} & $\mathrm{C} 16 \mathrm{H} 32 \mathrm{O} 2$ & Palmitic acid & $\downarrow * *$ & Krebs cycle \\
\hline
\end{tabular}

aThe levels of potential biomarkers were labeled with $(\uparrow)$ up-regulation and $(\downarrow)$ down-regulation $\left({ }^{*} p<0.05 ;{ }^{* *} p<0.01 ;{ }^{* * *} p<0.001\right)$. Change trend of $\mathrm{BDL}-3 \mathrm{~d}$ group vs control group.

bPC, Phosphatidylcholine.

'LysoPC, Lysophosphatidylcholine.
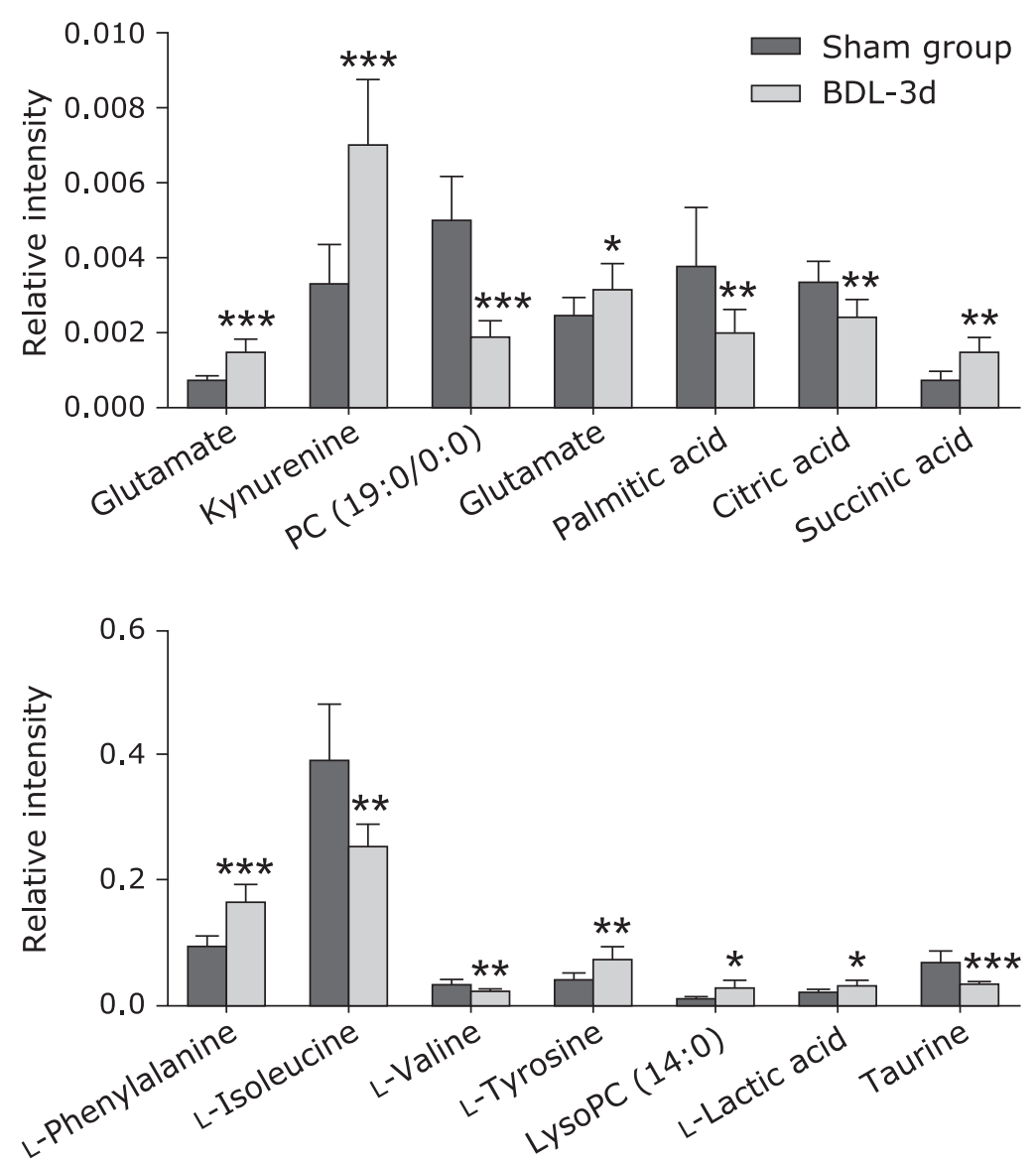

Fig. 4. RPLC-MS and HILIC-MS relative signal intensities for fourteen metabolites: sham control vs BDL. Data are expressed as mean \pm SD. Significant differences $(p<0.05)$ existed between sham and BDL for all the metabolites studied. ${ }^{*} p<0.05,{ }^{* *} p<0.01 ;{ }^{* * *} p<0.001$ vs sham control. 


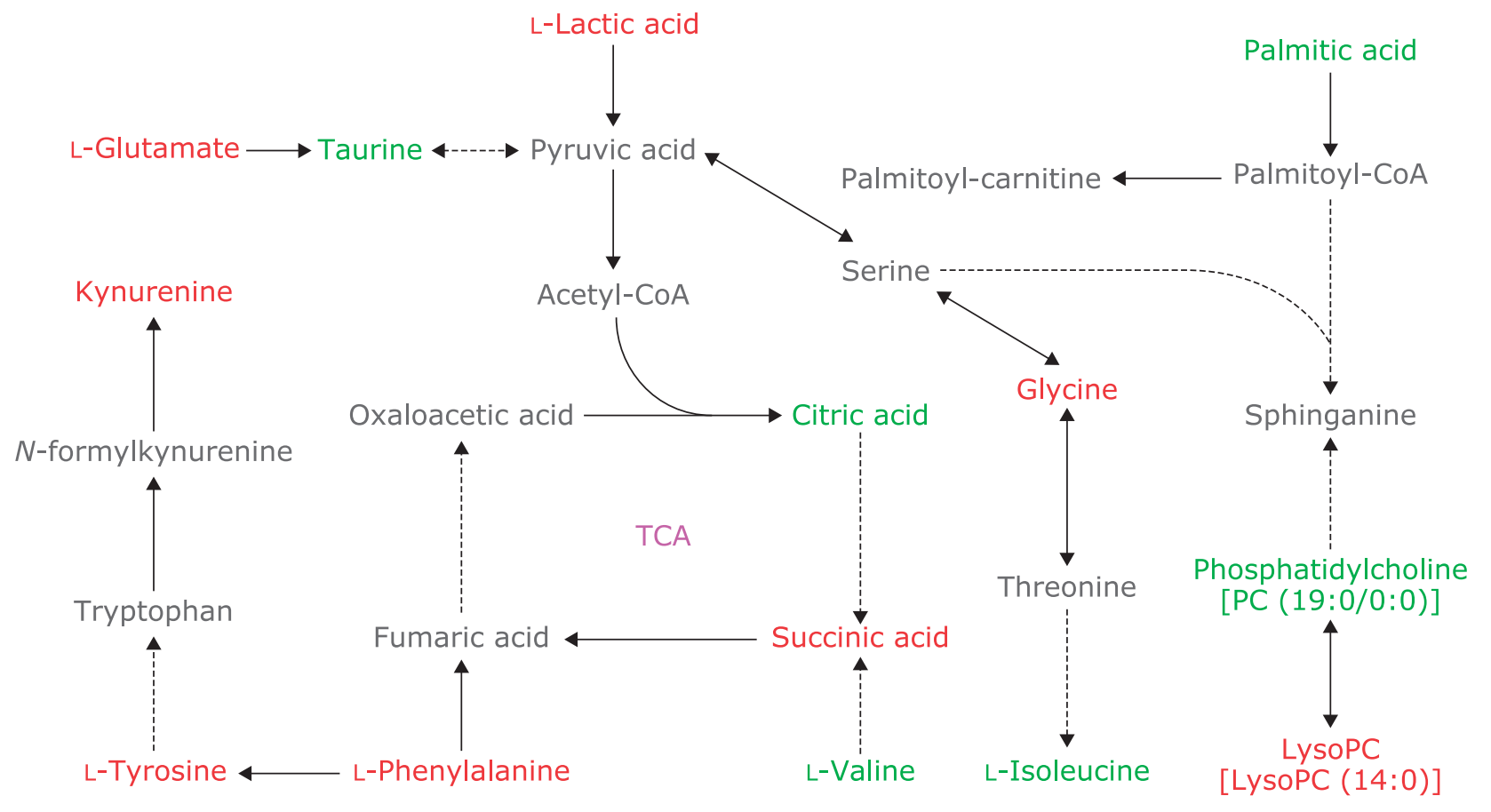

Fig. 5. Changes in metabolites and metabolic pathways upon BDL. Red: increase; green: decrease; black: no change. See online version figure.

Table 2. Primary indexes about oxidative stress in serum (mean $\pm \mathrm{SE})$

\begin{tabular}{lcclccc}
\hline Groups & MDA $(\mathrm{nmol} / \mathrm{ml})$ & \multicolumn{1}{c}{ SOD $(\mathrm{U} / \mathrm{ml})$} & GSH $(\mathrm{mg} / \mathrm{L})$ & \multicolumn{1}{c}{ GSH-PX } & GSSG $(\mu \mathrm{mol} / \mathrm{L})$ & T-AOC $(\mathrm{U} / \mathrm{ml})$ \\
\hline Sham3d group & $4.11 \pm 1.07$ & $156.03 \pm 6.17$ & $0.86 \pm 2.43$ & $271.47 \pm 23.94$ & $176.58 \pm 30.7$ & $11.52 \pm 0.85$ \\
BDL3d group & $8.97 \pm 0.83^{* *}$ & $109.84 \pm 9.78^{* *}$ & $3.21 \pm 0.85^{* *}$ & $189.80 \pm 15.49 * *$ & $187.63 \pm 19.94$ & $7.38 \pm 1.46^{*}$ \\
\hline
\end{tabular}

${ }^{*} p<0.05,{ }^{* *} p<0.01$ vs sham control.

excretion. ${ }^{(39)}$ Hence the reduction of GSH could cause cholestasis, increase lipid peroxidation of liver cells, lead to the accumulation of free radicals, and then aggravate hepatic injury induced by obstructive jaundice. GSH is synthesized from L-glutamic acid, Lcysteine and glycine and can be metabolized into cysteinylglycine, $\gamma$-glutamylcysteine, glycine and cysteine catalyzed by gamma glutamyl transferase $(\gamma-\mathrm{GT})$ and dipeptidase (DPT). ${ }^{(40)}$ The increase of L-glutamic and glycine in BDL rats indicates that GSH synthesis is attenuated and GSH catabolism is increased after BDL, which is in accordance with the GSH levels detected in the serum.

Phenylalanine hydroxylase $(\mathrm{PheH})$ catalyzes the hydroxylation of phenylalanine to tyrosine, a precursor for many hormones and neurotransmitters. Phenylalanine is also a precursor of important small molecules necessary for hormone synthesis. Increased plasma phenylalanine and tyrosine levels in BDL rats may underlie the nerve-endocrine dysfunction upon cholestasis. The synthesis of aromatic amino acids (phenylalanine and tyrosine) is usually located in microorganisms and plants, and in general, their glycolysis is carried out by anaerobic bacteria in the gut. So the change of the concentration of them may be involved in gut flora metabolism.

As an efficient endogenous antioxidant, taurine showed renoprotective effects in several model animals. ${ }^{(41)}$ Thus the decreased taurine in BDL rats may partly contribute to the oxidative stress and renal injury of obstructive jaundice.

Isoleucine is a branched chain amino acid (BCAA), and participates in glucose metabolism and provides energy through generating succinyl coenzyme A and acetyl coenzyme A from fatty acid beta oxidation. BCAA are important amino acids in energy metabolism, and particularly upon starvation. ${ }^{(42)}$ And their metabolic process may inhibit the consumption of liver and muscle glycogen, promote alanine-glucose cycle to reinforce the gluconeogenesis, hence is beneficial for energy supply in long time energy consumption. ${ }^{(43)}$ Thus the decrease of isoleucine and valine in the BDL rats suggest disturbance of three major nutrients such as sugar, fat, protein, immune function and energy metabolism in which the degradation of valine may be the main source of energy. The decrease of valine content may also have direct effect on raw materials and energy required for Krebs cycle, which was suppressed in BDL rats.

Upon liver damage, entry of amino acid into the blood, particularly aromatic amino acids, is increased. Liver dysfunction also decreases insulin inactivation and may participate in hyperinsulinemia. The intake of BCAA in skeletal muscle and adipose tissue is increased by insulin. As a consequence, the BCAA/AAA ratio is reduced which easily leads to hepatic encephalopathy. ${ }^{(44,45)}$

Glycolysis. An increase of lactic acid in cholestasis is concerned with the increase of lactic acid production and decreased clearance. It may be ascribed to the fast conversion of pyruvic acid to lactic acid induced by hypoxia and mitochondria energy metabolism disorder, which leads to the elevation of lactic acid generation. ${ }^{(46)}$ Besides, liver and kidney function decline in cholestasis may cause the decreased rate of lactic acid removal. A research showed that the elevation of peripheral blood lactic acid levels is the metabolic characteristic of rats with 
high risk of multiple organ failure and its degree elevation is proportional to the mortality, ${ }^{(47)}$ which is consistent with the feature of high complications and mortality in cholestasis. Furthermore, the abnormity of lactic acid levels suggest deficits in the overall energy metabolism. ${ }^{(48,49)}$ The elevation of lactic acid in model animals shows that liver and kidney injury, part hypoxia and other factors may make the membrane structure of visceral organs damaged and result in respiratory chain disorders as well as Krebs cycle and energy metabolic disorders in mitochondria.

Lipid metabolism. Lysophosphatidylcholine (LPC) is produced by hydrolysis of phosphatidylcholine (PC) by phospholipase A2. PC may act as serum antioxidant to prevent lipoprotein oxidation. ${ }^{(50)}$ Low-level PC may also play a part in an imbalanced oxidative stress. ${ }^{(51)}$ Many studies have shown that LPC can effectively induce asymmetric dimethylarginine (ADMA) ${ }^{(52-55)}$ and oxidative stress through activating the NADPH oxidase. ${ }^{(52,54,55)}$ Hence an increase of LPC and decrease of PC in the BDL rats in our study indicate the presence of phospholipids metabolic abnormality and an imbalance of oxidative environment. It is also conceivable that these variations are likely to ascribe to the activation of phospholipase A2. Besides, PC is a primary lipid component of biomembranes. ${ }^{(56)}$ LPC participates in a variety of cell signal transduction pathways in macrophages and $\mathrm{T}$ cells and especial receptors. ${ }^{(57-59)}$

Kynurenine (Kyn) is an important intermediate of tryptophan (Trp) metabolism, and is implicated in many human diseases. Kyn plays an important role in the adjustment of blood pressure and could activate cGMP signal transduction pathway. ${ }^{(60)}$ Kyn has immunosuppressive activity, and its generation also consumes Trp, which in turn is a essential amino acid of T cells. ${ }^{(61)} \mathrm{Kyn}$ levels is associated with the severity of chronic renal disease, also, serum Trp levels is negatively associated with uric acid and creatinine levels in patients. ${ }^{(62,63)}$ Pawlak et al. ${ }^{(64,65)}$ found that prominent alterations of the Kyn metabolism in peripheral blood in patients with chronic renal failure (CRF) resulted in the dramatic reductions of Trp levels and elevation of Kyn concentrations, and is directly related to higher levels of renal insufficiency. Kyn and its metabolites are important to proper functions of the central nervous system. Endogenous kynurenic substances are regulator of glutamatergic neurotransmission which may be involved in adjusting the brain function. Kyn is also the only known endogenous neural excitatory amino acids (EAA) receptor antagonist. The increase of Kyn in BDL rats may be relevant to the development of hypotension, renal failure, immunosuppression and may be a compensatory reaction of organism against the nerve function deficit in cholestasis.

Kreb's cycle. The increase of succinic acid, an intermediate in citric acid cycle, in BDL rats indicates hepatocytic mitochondria damage in cholestasis results in the Krebs cycle disorders, ${ }^{(66)}$ accumulation of intermediate and energy metabolism dysfunction. Under a normal state, stress should be put on aerobic respiration in cell energy metabolism. Palmitic acid and citric acid are both intermediates in the citric acid cycle, and their decline showed that cell aerobic metabolism decreased in rats with cholestasis which result in the buildup of acid metabolites in the body and there is a direct damage to cells. ${ }^{(67,68)} \mathrm{A}$ previous study showed that decreased function of the hepatic mitochondrion and the weakened citric acid cycle could decrease the intermediate metabolites levels. ${ }^{(69)}$

In summary, the 14 potential biomarkers are mainly associated with redox damage, hepatic injury, kidney injury, mitochondrial dysfunction, systemic endotoxemia, nerve function deficit, the disturbance in hemodynamic and immunosuppression. And oxidative stress may take part in the pathophysiological procedure of cholestasis.

Oxidative stress and cholestasis. We observed both a decrease in GSH and T-AOC as well as an increase in MDA blood level, consistent with a damaged redox balance. Both SOD and GSH-Px transform reactive free radicals into less reactive, or inactive species. In comparison to the sham control group, the BDL rats showed a significant decrease in activities of GSH-Px and SOD in the serum, validating the suppressed antioxidant efficiency in combating the cholestasis induced free radical damage.

There is also a growing body of evidence that obstructive jaundice leads to systemic oxidative stress, influencing all important organs like the liver, ${ }^{(8)}$ kidney, ${ }^{(9)}$ heart, ${ }^{(10)}$ brain,${ }^{(14)}$ lungs, ${ }^{(11)}$ intestine, ${ }^{(12)}$ and blood. ${ }^{(13)}$ The possible mechanism of increased oxidative stress in cholestasis is complicated. Evidence has accumulated that oxidative stress is cholestatic in essence recently. ${ }^{(70)}$ Schmitt et al. ${ }^{(71)}$ and Kawai et al. ${ }^{(72)}$ found a fast decline in bile flow, causing a complete cholestasis, after exposing rat livers with the pro-oxidant tert-butylhydroperoxide (tBuOOH). And a role for oxidative stress as a principal etiology and/or a worsening element has been supported in extrahepatic cholestasis caused by bile duct ligation. ${ }^{(70,73,74)}$ Generally speaking, oxidative stress needs a combination of increased reactive oxygen species (ROS) formation and damage of antioxidant systems to cause a relevant influence. Several researches have shown that free oxygen radicals participate in the pathogenesis of the cholestatic syndrome. ${ }^{(75,76)}$ Content of ROS and toxic degradative products of lipid peroxidation increase in several models of clinical and experimental cholestasis. ${ }^{(10,75)}$

Mitochondria are involved in several vital cellular processes such as energy production, apoptosis, fatty acid metabolism, pyrimidine biosynthesis and calcium homeostasis. The foremost cellular sources of ROS are mitochondria. ${ }^{(77)}$ Mitochondria are likely to the source of ROS in liver cells exposed to pathologic concentrations of bile acids. ${ }^{(78)}$ ROS are over-produced within cells when oxidative stress is generated and mitochondrial function is deficient. ${ }^{(79)}$ Moreover, hepatic injury and fibrosis decreased followed by exposure to mitochondrial Mn-SOD; on the contrary, expression of cytosolic $\mathrm{Cu} / \mathrm{Zn}$-SOD was primarily invalid. ${ }^{(80)}$ Put together, mitochondrial ROS are likely to be vital for liver damage in cholestasis.

Oxidant stress plays an vital role in the mechanisms of liver injury and hepatocyte apoptosis during cholestasis. ${ }^{(10)}$ The agents that induce apoptosis include both oxidants and stimulators of cellular oxidative metabolism. ${ }^{(81)}$ Free radicals, specifically ROS, are common mediators of apoptosis. Reactive oxygen intermediates are implicated in the induction of hepatocyte apoptosis in cholestasis. ${ }^{(10,82,83)}$ Oxidative stress was considered by some as an unlikely primary contributor to the development of hepatic injury during cholestasis at early phase. ${ }^{(73)}$ And although in vitro studies have shown that bile acids cause hepatocyte apoptosis by mechanisms that rely on ROS, proofs from in vivo researches suggest that this mechanism is of limited importance for liver damage during cholestasis. ${ }^{(73)}$

Oxidative stress is also related to serious complications like renal dysfunction in cholestasis. ${ }^{(84)}$ It plays an important role in kidney dysfunction of obstructive jaundice via the formation of several vasoactive mediators and direct effect on glomerular microcirculation through inducing the contraction of mesangial cells, and in consequence reducing the glomerular capillary ultrafiltration coefficient. ${ }^{(85)}$

Obstructive jaundice leads to development of oxidative injury. ${ }^{(86)}$ This is often concerned with the change of the nutrient material in the intestine and damage of intestinal barrier. ${ }^{(87)}$ Intestinal barrier dysfunction causes remarkable increases in intestinal permeability and bacterial translocation, which results in systemic endotoxemia and inflammatory response and afterwards intestinal barrier dysfunction will grow worse. ${ }^{(88,89)}$ Oxidative stress and enhanced concentration of proinflammatory cytokines like tumor necrosis factor and interleukin- 6 have also been implicated. ${ }^{(90,91)}$ For these reasons, protecting the integrity of the 
intestinal barrier and preventing oxidative damage are important goals in the management of endotoxemia in obstructive jaundice.

Besides that, a study also describes increased oxidative stress in central nervous system that is likely to explain associated complications during cholestasis. ${ }^{(92)}$ Several researches have also narrated the induction of oxidative stress to neurological disorders. ${ }^{(93,94)}$ Moreover, scientists conclude that obstructive jaundice may aggravate oxidative stress in the brain and free radicals may be concerned with the pathogenesis of hepatic encephalopathy. ${ }^{(14,95)}$

\section{Conclusion}

In the current study, we developed a metabonomic methods based on combination of RPLC-Q-TOF MS and HILIC-Q-TOF $\mathrm{MS}$, and examined global metabolomics changes after BDL in

\section{References}

1 Pauli-Magnus C, Meier PJ. Hepatocellular transporters and cholestasis. $J$ Clin Gastroenterol 2005; 39: S103-S110.

2 Wadei HM, Mai ML, Ahsan N, Gonwa TA. Hepatorenal syndrome: pathophysiology and management. Clin J Am Soc Nephrol 2006; 1: 1066-1079.

3 Furukawa Y. Histological changes in the brain due to experimental obstructive jaundice. Nihon Geka Gakkai Zasshi 1991; 92: 37-45 (in Japanese).

4 Papadopoulos V, Filippou D, Manolis E, Mimidis K. Haemostasis impairment in patients with obstructive jaundice. J Gastrointestin Liver Dis 2007; 16: $177-186$.

5 Nehéz L, Andersson R. Compromise of immune function in obstructive jaundice. Eur J Surg 2002; 168: 315-328.

6 Papakostas C, Bezirtzoglou E, Pitiakoudis M, Polychronidis A, Simopoulos C. Endotoxinemia in the portal and the systemic circulation in obstructive jaundice. Clin Exp Med 2003; 3: 124-128.

7 Pain JA, Bailey ME. Measurement of operative plasma endotoxin levels in jaundiced and non-jaundiced patients. Eur Surg Res 1987; 19: 207-216.

8 Assimakopoulos SF, Vagianos CE, Zervoudakis G, et al. Gut regulatory peptides bombesin and neurotensin reduce hepatic oxidative stress and histological alterations in bile duct ligated rats. Regul Pept 2004; 120: 185-193.

9 Ara C, Karabulut AB, Kirimlioglu H, et al. Protective effect of resveratrol against renal oxidative stress in cholestasis. Ren Fail 2005; 27: 435-440.

10 Ljubuncic P, Tanne Z, Bomzon A. Evidence of a systemic phenomenon for oxidative stress in cholestatic liver disease. Gut 2000; 47: 710-716.

11 Tieppo J, Vercelino R, Dias AS, et al. Evaluation of the protective effects of quercetin in the hepatopulmonary syndrome. Food Chem Toxicol 2007; 45: 1140-1146.

12 Assimakopoulos SF, Vagianos CE, Patsoukis N, Georgiou C, Nikolopoulou $\mathrm{V}$, Scopa CD. Evidence for intestinal oxidative stress in obstructive jaundiceinduced gut barrier dysfunction in rats. Acta Physiol Scand 2004; 180: 177 185.

13 Tsai LY, Lee KT, Liu TZ. Evidence for accelerated generation of hydroxyl radicals in experimental obstructive jaundice of rats. Free Radic Biol Med 1998; 24: 732-737.

14 Chroni E, Patsoukis N, Karageorgos N, Konstantinou D, Georgiou C. Brain oxidative stress induced by obstructive jaundice in rats. J Neuropathol Exp Neurol 2006; 65: 193-198.

15 Brindle JT, Antti H, Holmes E, et al. Rapid and noninvasive diagnosis of the presence and severity of coronary heart disease using 1H-NMR-based metabonomics. Nat Med 2002; 8: 1439-1444.

16 Ganti S, Taylor SL, Abu Aboud. Kidney tumor biomarkers revealed by simultaneous multiple matrix metabolomics analysis. Cancer Res 2012; 72: 3471-3479.

17 Sreekumar A, Poisson LM, Rajendiran TM, et al. Metabolomic profiles delineate potential role for sarcosine in prostate cancer progression. Nature 2009; 457: 910-914.

18 Nicholson JK, Lindon JC, Holmes E. 'Metabonomics': understanding the metabolic responses of living systems to pathophysiological stimuli via multivariate statistical analysis of biological $\mathrm{nmr}$ spectroscopic data. Xenobiotica 1999; 29: 1181-1189.

19 Wang X, Sun H, Zhang A, Sun W, Wang P, Wang Z. Potential role of metabolomics apporoaches in the area of traditional chinese medicine: as rats. We identified 14 differentially expressed metabolites, belonging mainly to amino acid metabolism, lipid metabolism, glycolysis and Krebs cycle. And the serum biochemical data showed that cholestasis was associated with oxidative stress. Verification in human subjects is being planned.

\section{Acknowledgments}

This study was supported by the National Natural Science Foundation of China (81170427).

\section{Conflict of Interest}

The authors declare that they have no conflict of interests with biotech companies, financial foundations, or any other third parties.

pillars of the bridge between chinese and western medicine. J Pharm Biomed Anal 2011; 55: 859-868.

20 Zhang T, Watson DG, Wang L, et al. Application of holistic liquid chromatography-high resolution mass spectrometry based urinary metabolomics for prostate cancer detection and biomarker discovery. PLoS One 2013; 8: e65880.

21 Castro C, Krumsiek J, Lehrbach NJ, Murfitt SA, Miska EA, Griffin JL. A study of caenorhabditis elegans DAF-2 mutants by metabolomics and differential correlation networks. Mol Biosyst 2013; 9: 1632-1642.

$22 \mathrm{Xu} \mathrm{J}$, Liu C, Cai S, et al. Metabolomic profilings of urine and serum from high fat-fed rats via $1 \mathrm{H}$ NMR spectroscopy and pattern recognition. Appl Biochem Biotechnol 2013; 169: 1250-1261.

23 Wang X, Yang B, Sun H, Zhang A. Pattern recognition approaches and computational systems tools for ultra performance liquid chromatographymass spectrometry-based comprehensive metabolomic profiling and pathways analysis of biological data sets. Anal Chem 2012; 84: 428-439.

24 Yang B, Zhang A, Sun H, et al. Metabolomic study of insomnia and intervention effects of suanzaoren decoction using ultra-performance liquidchromatography/electrospray-ionization synapt high-definition mass spectrometry. J Pharm Biomed Anal 2012; 58: 113-124.

25 OuYang D, Xu J, Huang H, Chen Z. Metabolomic profiling of serum from human pancreatic cancer patients using $1 \mathrm{H}$ NMR spectroscopy and principal component analysis. Appl Biochem Biotechnol 2011; 165: 148-154.

26 Psychogios N, Hau DD, Peng J, et al. The human serum metabolome. PLoS One 2011; 6: e16957.

27 Arjmand M, Kompany-Zareh M, Vasighi M, Parvizzadeh N, Zamani Z, Nazgooei F. Nuclear magnetic resonance-based screening of thalassemia and quantification of some hematological parameters using chemometric methods. Talanta 2010; 81: 1229-1236.

28 Wilson ID, Nicholson JK, Castro-Perez J, et al. High resolution "ultra performance" liquid chromatography coupled to oa-tof mass spectrometry as a tool for differential metabolic pathway profiling in functional genomic studies. $J$ Proteome Res 2005; 4: 591-598.

29 Lenz EM, Wilson ID. Analytical strategies in metabonomics. J Proteome Res 2007; 6: 443-458.

30 Yang J, Xu G, Hong Q, et al. Discrimination of type 2 diabetic patients from healthy controls by using metabonomics method based on their serum fatty acid profiles. J Chromatogr B Analyt Technol Biomed Life Sci 2004; 813: $53-$ 58.

31 Zakaria FA. Effect of oxygen derived free radicals and glycine on sodiumpotassium adenosine triphosphatase in the basolateral membrane of the kidney in ischemia-reperfusion. Saudi Med J 2002; 23: 1380-1385.

32 Grotz MR, Pape HC, van Griensven. Glycine reduces the inflammatory response and organ damage in a two-hit sepsis model in rats. Shock 2001; 16: 116-121.

33 Zhong Z, Li X, Yamashina S, et al. Cyclosporin A causes a hypermetabolic state and hypoxia in the liver: Prevention by dietary glycine. J Pharmacol Exp Ther 2001; 299: 858-865.

34 Qu W, Ikejima K, Zhong Z, Waalkes MP, Thurman RG. Glycine blocks the increase in intracellular free $\mathrm{Ca}^{2+}$ due to vasoactive mediators in hepatic parenchymal cells. Am J Physiol Gastrointest Liver Physiol 2002; 283 
G1249-G1256.

35 Ikejima K, Iimuro Y, Forman DT, Thurman RG. A diet containing glycine improves survival in endotoxin shock in the rat. Am J Physiol 1996; 271: G97-G103.

36 DeLeve LD, Kaplowitz N. Glutathione metabolism and its role in hepatotoxicity. Pharmacol Ther 1991; 52: 287-305.

37 Shaw S, Herbert V, Colman N, Jayatilleke E. Effect of ethanol-generated free radicals on gastric intrinsic factor and glutathione. Alcohol 1990; 7: 153-157.

38 Arteel GE. Oxidants and antioxidants in alcohol-induced liver disease. Gastroenterology 2003; 124: 778-790.

39 Ballatori N, Truong AT. Glutathione as a primary osmotic driving force in hepatic bile formation. Am J Physiol 1992; 263: G617-G624.

40 Abbott WA, Meister A. Intrahepatic transport and utilization of biliary glutathione and its metabolites. Proc Natl Acad Sci US A 1986; 83: 1246-1250.

41 Wang L, Zhang L, Yu Y, Wang Y, Niu N. The protective effects of taurine against early renal injury in STZ-induced diabetic rats, correlated with inhibition of renal LOX-1-mediated ICAM-1 expression. Ren Fail 2008; 30: 763771.

42 Ji LL, Miller RH, Nagle FJ, Lardy HA, Stratman FW. Amino acid metabolism during exercise in trained rats: the potential role of carnitine in the metabolic fate of branched-chain amino acids. Metabolism 1987; 36: 748752.

43 Shimomura Y, Murakami T, Nakai N, et al. Suppression of glycogen consumption during acute exercise by dietary branched-chain amino acids in rats. J Nutr Sci Vitaminol (Tokyo) 2000; 46: 71-77.

44 Morgan MY, Milsom JP, Sherlock S. Plasma ratio of valine, leucine and isoleucine to phenylalanine and tyrosine in liver disease. Gut 1978; 19: 10681073.

45 Morgan MY, Marshall AW, Milsom JP, Sherlock S. Plasma amino-acid patterns in liver disease. Gut 1982; 23: 362-370.

46 Brealey D, Karyampudi S, Jacques TS, et al. Mitochondrial dysfunction in a long-term rodent model of sepsis and organ failure. Am J Physiol Regul Integr Comp Physiol 2004; 286: R491-R497.

47 Joynt GM, Lipman J, Gomersall CD, Tan I, Scribante J. Gastric intramucosal ph and blood lactate in severe sepsis. Anaesthesia 1997; 52: 726-732.

48 Constantinou MA, Theocharis SE, Mikros E. Application of metabonomics on an experimental model of fibrosis and cirrhosis induced by thioacetamide in rats. Toxicol Appl Pharmacol 2007; 218: 11-19.

49 Nicholson JK, Wilson ID. Opinion: understanding 'global' systems biology: metabonomics and the continuum of metabolism. Nat Rev Drug Discov 2003; 2: 668-676.

50 Wallner S, Schmitz G. Plasmalogens the neglected regulatory and scavenging lipid species. Chem Phys Lipids 2011; 164: 573-589.

51 Treede I, Braun A, Sparla R, et al. Anti-inflammatory effects of phosphatidylcholine. J Biol Chem 2007; 282: 27155-27164.

52 Abid MR, Kachra Z, Spokes KC, Aird WC. Nadph oxidase activity is required for endothelial cell proliferation and migration. FEBS Lett 2000; 486: $252-256$.

53 Jiang DJ, Jiang JL, Tan GS, Du YH, Xu KP, Li YJ. Protective effects of daviditin A against endothelial damage induced by lysophosphatidylcholine. Naunyn Schmiedebergs Arch Pharmacol 2003; 367: 600-606.

54 Takeshita S, Inoue N, Gao D, et al. Lysophosphatidylcholine enhances superoxide anions production via endothelial nadh/nadph oxidase. $J$ Atheroscler Thromb 2000; 7: 238-246.

55 Inoue N, Takeshita S, Gao D, et al. Lysophosphatidylcholine increases the secretion of matrix metalloproteinase 2 through the activation of nadh/nadph oxidase in cultured aortic endothelial cells. Atherosclerosis $2001 ; 155$ : 45-52.

56 Wright MM, Howe AG, Zaremberg V. Cell membranes and apoptosis: role of cardiolipin, phosphatidylcholine, and anticancer lipid analogues. Biochem Cell Biol 2004; 82: 18-26.

57 Duong CQ, Bared SM, Abu-Khader A, Buechler C, Schmitz A, Schmitz G. Expression of the lysophospholipid receptor family and investigation of lysophospholipid-mediated responses in human macrophages. Biochim Biophys Acta 2004; 1682: 112-119.

58 Kabarowski JH. G2A and LPC: regulatory functions in immunity. Prostaglandins Other Lipid Mediat 2009; 89: 73-81.

59 Oestvang J, Johansen B. PhospholipaseA2: a key regulator of inflammatory signalling and a connector to fibrosis development in atherosclerosis. Biochim Biophys Acta 2006; 1761: 1309-1316.

60 Guo S, Vecsei L, Ashina M. The L-kynurenine signalling pathway in trigeminal pain processing: a potential therapeutic target in migraine? Cephalalgia 2011; 31: 1029-1038.
61 Heitger A. Regulation of expression and function of IDO in human dendritic cells. Curr Med Chem 2011; 18: 2222-2233.

62 Myśliwiec P, Myśliwiec H, Pawlak D, Dadan J, Buczko W, Pawlak K. Tryptophan and its metabolites in renal allograft recipients. Przegl Lek 2009; 66: 115-118.

63 Schefold JC, Zeden JP, Fotopoulou C, et al. Increased indoleamine 2,3dioxygenase (ido) activity and elevated serum levels of tryptophan catabolites in patients with chronic kidney disease: A possible link between chronic inflammation and uraemic symptoms. Nephrol Dial Transplant 2009; 24: 1901-1908.

64 Pawlak K, Kowalewska A, Mysliwiec M, Pawlak D. Kynurenine and its metabolites--kynurenic acid and anthranilic acid are associated with soluble endothelial adhesion molecules and oxidative status in patients with chronic kidney disease. Am J Med Sci 2009; 338: 293-300.

65 Pawlak D, Tankiewicz A, Matys T, Buczko W. Peripheral distribution of kynurenine metabolites and activity of kynurenine pathway enzymes in renal failure. J Physiol Pharmacol 2003; 54: 175-189.

66 Bubber P, Hartounian V, Gibson GE, Blass JP. Abnormalities in the tricarboxylic acid (TCA) cycle in the brains of schizophrenia patients. Eur Neuropsychopharmacol 2011; 21: 254-260.

67 Psihogios NG, Gazi IF, Elisaf MS, Seferiadis KI, Bairaktari ET. Genderrelated and age-related urinalysis of healthy subjects by NMR-based metabonomics. NMR Biomed 2008; 21: 195-207.

68 Schurr A, Payne RS, Miller JJ, Tseng MT, Rigor BM. Blockade of lactate transport exacerbates delayed neuronal damage in a rat model of cerebral ischemia. Brain Res 2001; 895: 268-272.

69 Schieke SM, Finkel T. Mitochondrial signaling, TOR, and life span. Biol Chem 2006; 387: 1357-1361.

70 Roma MG, Sanchez Pozzi. Oxidative stress: a radical way to stop making bile. Ann Hepatol 2008; 7: 16-33.

71 Schmitt M, Kubitz R, Wettstein M, vom Dahl, Haussinger D. Retrieval of the mrp2 gene encoded conjugate export pump from the canalicular membrane contributes to cholestasis induced by tert-butyl hydroperoxide and chlorodinitrobenzene. Biol Chem 2000; 381: 487-495.

72 Kawai S, Arai T, Yokoyama Y, Nagino M, Nimura Y. Free oxygen radicals reduce bile flow in rats via an intracellular cyclic amp-dependent mechanism. J Gastroenterol Hepatol 2007; 22: 429-435.

73 Copple BL, Jaeschke H, Klaassen CD. Oxidative stress and the pathogenesis of cholestasis. Semin Liver Dis 2010; 30: 195-204.

74 Pérez LM, Milkiewicz P, Elias E, Coleman R, Sánchez Pozzi, Roma MG. Oxidative stress induces internalization of the bile salt export pump, Bsep, and bile salt secretory failure in isolated rat hepatocyte couplets: a role for protein kinase $\mathrm{C}$ and prevention by protein kinase A. Toxicol Sci 2006; 91: $150-158$.

75 Barón V, Muriel P. Role of glutathione, lipid peroxidation and antioxidants on acute bile-duct obstruction in the rat. Biochim Biophys Acta 1999; 1472: 173-180.

76 Holt S, Marley R, Fernando B, et al. Acute cholestasis-induced renal failure: effects of antioxidants and ligands for the thromboxane A2 receptor. Kidney Int 1999; 55: 271-277.

77 Murphy MP. How mitochondria produce reactive oxygen species. Biochem J 2009; 417: 1-13.

78 Sokol RJ, Winklhofer-Roob BM, Devereaux MW, McKim JM, Jr. Generation of hydroperoxides in isolated rat hepatocytes and hepatic mitochondria exposed to hydrophobic bile acids. Gastroenterology 1995; 109: 1249-1256.

79 Therade-Matharan S, Laemmel E, Duranteau J, Vicaut E. Reoxygenation after hypoxia and glucose depletion causes reactive oxygen species production by mitochondria in HUVEC. Am J Physiol Regul Integr Comp Physiol 2004; 287: R1037-R1043.

80 Zhong Z, Froh M, Wheeler MD, Smutney O, Lehmann TG, Thurman RG. Viral gene delivery of superoxide dismutase attenuates experimental cholestasis-induced liver fibrosis in the rat. Gene Ther 2002; 9: 183-191.

81 Haddad JJ. Redox and oxidant-mediated regulation of apoptosis signaling pathways: immuno-pharmaco-redox conception of oxidative siege versus cell death commitment. Int Immunopharmacol 2004; 4: 475-493.

82 Czaja MJ. Induction and regulation of hepatocyte apoptosis by oxidative stress. Antioxid Redox Signal 2002; 4: 759-767.

83 Shafaroodi H, Ebrahimi F, Moezi L, et al. Cholestasis induces apoptosis in mice cardiac cells: the possible role of nitric oxide and oxidative stress. Liver Int 2010; 30: 898-905.

84 Cruz A, Padillo FJ, Túnez I, et al. Melatonin protects against renal oxidative stress after obstructive jaundice in rats. Eur J Pharmacol 2001; 425: 135-139. 
85 Bomzon A, Holt S, Moore K. Bile acids, oxidative stress, and renal function in biliary obstruction. Semin Nephrol 1997; 17: 549-562.

86 Kountouras J, Billing BH, Scheuer PJ. Prolonged bile duct obstruction: a new experimental model for cirrhosis in the rat. Br J Exp Pathol 1984; 65: 305-311.

87 Selberg O, Böttcher J, Tusch G, Pichlmayr R, Henkel E, Müller MJ. Identification of high- and low-risk patients before liver transplantation: a prospective cohort study of nutritional and metabolic parameters in 150 patients. Hepatology 1997; 25: 652-657.

88 McKay DM, Baird AW. Cytokine regulation of epithelial permeability and ion transport. Gut 1999; 44: 283-289.

89 Pérez del Pulgar S, Pizcueta P, Engel P, Bosch J. Enhanced monocyte activation and hepatotoxicity in response to endotoxin in portal hypertension. $J$ Hepatol 2000; 32: 25-31.

90 Lechner AJ, Velasquez A, Knudsen KR, Johanns CA, Tracy TF, Jr, Matuschak GM. Cholestatic liver injury increases circulating TNF-alpha and
IL-6 and mortality after Escherichia coli endotoxemia. Am J Respir Crit Care Med 1998; 157: 1550-1558.

91 Liu TZ, Lee KT, Chern CL, Cheng JT, Stern A, Tsai LY. Free radicaltriggered hepatic injury of experimental obstructive jaundice of rats involves overproduction of proinflammatory cytokines and enhanced activation of nuclear factor kappab. Ann Clin Lab Sci 2001; 31: 383-390.

92 Cruz A, Túnez I, Martínez R, et al. Melatonin prevents brain oxidative stress induced by obstructive jaundice in rats. J Neurosci Res $2007 ; \mathbf{8 5}$ : 3652-3656.

93 Facheris M, Beretta S, Ferrarese C. Peripheral markers of oxidative stress and excitotoxicity in neurodegenerative disorders: tools for diagnosis and therapy? J Alzheimers Dis 2004; 6: 177-184.

94 Cherubini A, Ruggiero C, Polidori MC, Mecocci P. Potential markers of oxidative stress in stroke. Free Radic Biol Med 2005; 39: 841-852.

95 Norenberg MD, Jayakumar AR, Rama Rao. Oxidative stress in the pathogenesis of hepatic encephalopathy. Metab Brain Dis 2004; 19: 313-329. 